\title{
Biased Intensity Judgements of Visceral Sensations After Learning to Fear Visceral Stimuli: A Drift Diffusion Approach
}

\author{
Jonas Zaman, ${ }^{\star}$ Victoria J. Madden, ${ }^{\dagger}$ Julie Iven, ${ }^{\ddagger}$ Katja Wiech, ${ }^{\S},{ }^{\Uparrow}$ Nathalie Weltens, ${ }^{\star}$ \\ Huynh Giao Ly, ${ }^{\ddagger}$ Johan W. S. Vlaeyen, ${ }^{*}, \|$ Lukas Van Oudenhove, ${ }^{\ddagger}{ }^{* *}$ and Ilse Van Diest* \\ *Health Psychology, Faculty of Psychology and Educational Sciences, ${ }^{\ddagger}$ Laboratory for Brain-Gut Axis Studies (LaBGAS), \\ Translational Research Centre for Gastrointestinal Disorders (TARGID), Department of Clinical and Experimental \\ Medicine, and **Consultation-Liaison Psychiatry, University Psychiatric Centre, KU Leuven, Leuven, Belgium. \\ 'Sansom Institute for Health Research, The University of South Australia, Adelaide, Australia. \\ ${ }^{\S}$ Centre for Functional Magnetic Resonance Imaging of the Brain (FMRIB), University of Oxford, John Radcliffe \\ Hospital, Oxford, United Kingdom. \\ "Nuffield Department of Clinical Neurosciences, Nuffield Division Anaesthetics, University of Oxford, John Radcliffe \\ Hospital, Oxford, United Kingdom. \\ "Department Clinical Psychological Science, Maastricht University, Maastricht, The Netherlands.
}

\begin{abstract}
A growing body of research has identified fear of visceral sensations as a potential mechanism in the development and maintenance of visceral pain disorders. However, the extent to which such learned fear affects visceroception remains unclear. To address this question, we used a differential fear conditioning paradigm with nonpainful esophageal balloon distensions of 2 different intensities as conditioning stimuli (CSs). The experiment comprised of preacquisition, acquisition, and postacquisition phases during which participants categorized the CSs with respect to their intensity. The CS + was always followed by a painful electrical stimulus (unconditioned stimulus) during the acquisition phase and in $60 \%$ of the trials during postacquisition. The second stimulus (CS-) was never associated with pain. Analyses of galvanic skin and startle eyeblink responses as physiological markers of successful conditioning showed increased fear responses to the CS + compared with the CS-, but only in the group with the low-intensity stimulus as CS+. Computational modeling of response times and response accuracies revealed that differential fear learning affected perceptual decision-making about the intensities of visceral sensations such that sensations were more likely to be categorized as more intense. These results suggest that associative learning might indeed contribute to visceral hypersensitivity in functional gastrointestinal disorders.

Perspective: This study shows that associative fear learning biases intensity judgements of visceral sensations toward perceiving such sensations as more intense. Learning-induced alterations in visceroception might therefore contribute to the development or maintenance of visceral pain.
\end{abstract}

(c) 2017 by the American Pain Society

Key words: Interoceptive fear learning, perceptual decision-making, drift diffusion, visceral intensity perception, differential conditioning.

Received December 23, 2016; Revised April 5, 2017; Accepted April 23, 2017.

L.V.O. and I.V.D. contributed equally to this work as senior authors.

J.Z. was supported by a Personal Doctoral Mandate grant of the KU Leuven Research Council, the Centre for Excellence of Generalization Research in III Health and Psychopathology (PF/10/005, KU Leuven), and the "Asthenes" long-term structural funding (METH/15/011) - Methusalem grant by the Flemish Government. K.W. was supported by a grant of the Medical Research Council, United Kingdom. J.W.S.V. was supported by an Odysseus grant provided by the Research Foundation, Flanders, Belgium. J.W.S.V. and L.V.O. are Research Professors of the KU Leuven Special Research Fund ("Bijzonder Onderzoeksfonds"). N.W. is supported by a research grant funded by the Research Foundation, Flanders, Belgium (G085013N). H.G.L. is supported by a postdoctoral fellow- ship of the KU Leuven Special Research Fund. V.J.M. was supported by the Oppenheimer Memorial Trust (South Africa).

The authors have no conflicts of interest to declare.

Supplementary data accompanying this article are available online at www.jpain.org and www.sciencedirect.com.

Address reprint requests to Jonas Zaman, MSc, PhD, Health Psychology, KU Leuven, Tiensestraat 102, 3080 Leuven, Belgium. E-mail: jonas. zaman@kuleuven.be

$1526-5900 / \$ 36.00$

(c) 2017 by the American Pain Society

http://dx.doi.org/10.1016/j.jpain.2017.04.011 
$\mathrm{F}$ unctional gastrointestinal disorders, including irritable bowel syndrome, are characterized by frequent episodes of visceral pain. Although chronic visceral pain has tremendous direct and indirect societal costs ${ }^{19}$ and disabling effects, ${ }^{8,33}$ our understanding of its etiology is limited. However, recent work has implicated psychological processes or states such as gastrointestinal symptom-specific anxiety (GSA) in its pathogenesis. ${ }^{11,30,35}$ GSA comprises cognitive as well as affective responses that are attributed to fear of gastrointestinal sensations and associated contexts, ${ }^{15}$ and is assumed to develop through associative learning. ${ }^{7}$ In an experimental context, such processes are often studied using a Pavlovian conditioning paradigm ${ }^{22}$ in which a 'neutral' stimulus (conditioned stimulus [CS], eg, picture) elicits a preparatory fear response after it has preceded an aversive stimulus (unconditioned stimulus [US], eg, pain). Interoceptive $\mathrm{CSs}^{7,20,21,28,42}$ closely resemble clinical situations in which patients show preoccupation with and fear of such sensations. ${ }^{15}$ We recently showed that nonpainful visceral CSs can trigger anticipatory fear responses after being paired with a painful electrical US, ${ }^{44}$ indicating that interoceptive sensations can act as predictors of aversive outcomes or as safety cues. ${ }^{20,21,28}$ In the same study, perceptual habituation was conspicuously absent, ${ }^{44}$ suggesting a learning-induced change in visceroception that corroborated other studies in which fear learning influenced interoception ${ }^{43,44}$ and altered perceptual thresholds. ${ }^{1}$

We recently proposed that learning to fear interoceptive stimuli could induce a bias toward perceiving visceral sensations as more intense, aversive, or even painful. ${ }^{43}$ In this framework, visceroception is considered to be an inferential process during which sensory input is interpreted on the basis of previous knowledge. This sort of perceptual decision-making process can be investigated using computational models, such as the drift diffusion model (DDM), that use response time (RT) distributions of correct and incorrect responses during a 2alternative forced-choice task as input. The DDM assumes that evidence (ie, sensory input) favoring each possible response is integrated over time and that a decision is made when sufficient evidence has accumulated in favor of one alternative over the other ${ }^{4}$ (Fig 1). The alternative responses are represented by 2 orthogonal decision thresholds, which have to be reached to make a decision. The point at which this accumulation of evidence begins depends on previous knowledge, and can therefore be shifted toward 1 of the 2 decision thresholds, such that less evidence is required to reach that particular decision threshold (for more details see Ratcliff and McKoon, ${ }^{24}$ and Wiecki et al $^{40}$; Fig 1). These models have been successfully applied in a wide range of research to investigate, for instance, the influence of stimulus quality, expectations, or task instruction on decision-making. 6,18,23,39 The current study used DDM to explore how fear learning influences the perceptual decision-making process that underlies healthy volunteers' judgements about the intensity of visceral sensations. We used a differential conditioning paradigm with 2 nonpainful esophageal balloon distensions of different intensities (low- and high-intensity stimuli, $S_{L}$ and $S_{H}$, respectively) as CS+ (predictive of the US) and CS- (predictive of no US). Throughout the experiment, participants categorized the CSs as high or low, and accuracy and $R T$ s were used to estimate the parameters of the DDM. During preacquisition, only CSs were administered (baseline). From acquisition onward, the CS + was paired with a painful electrical US. Fear was indexed using the potentiated startle eyeblink reflex and galvanic skin response (GSR). ${ }^{17}$ We expected a potentiated startle eyeblink reflex from preacquisition to acquisition for the $\mathrm{CS}+$ relative to the $\mathrm{CS}-$, and less habituation of the GSR to the CS+ than to the CS- (ie, differential fear learning). Furthermore, in the DDM, we expected differential fear learning to result in a shift in starting point toward the CS+ threshold.

\section{Methods}

\section{Participants}

Of the 86 healthy volunteers recruited, 60 participants completed the entire experiment (33 female, 27 male, mean age $=21.4$ [SD $=2.2$ years). Reasons for early termination were: 1) intolerance of the esophageal probe $(n=9), 2$ ) esophageal hypersensitivity $(n=6)$, or 3 ) esophageal hyposensitivity $(n=7)$ to the balloon distensions, and 4) technical failure $(n=4)$ (for more details see Esophageal Stimulation). Exclusion criteria were a history or current state of psychiatric, gastrointestinal, neurological, cardiovascular, or chronic pain disorder or abdominal or thoracic surgery. All participants provided their written informed consent. The study was approved by the Medical Ethical Committee of the University Hospitals Leuven. Participants received a monetary compensation of $€ 50$.

\section{Esophageal Stimulation}

A 2.7-mm pediatric feeding tube (TR-2008; Pennine Healthcare, Derby, United Kingdom), with a deflated custom-made medical balloon (25-mm; Medasil Surgical Ltd, Leeds, United Kingdom) attached to its end, was inserted intranasally, with the balloon being positioned in the distal, autonomically innervated part of the esophagus, approximately $35 \mathrm{~cm}$ from the nostril. The extracorporeal part of the tube was draped over the ear and attached with adhesive tape to prevent it from moving. This procedure has been used extensively in previous research. ${ }^{37,44}$ A custom-made inflation pump (Medical Physics department, Salford Royal NHS Foundation Trust, Manchester, United Kingdom) with a digital display was used to inflate and deflate the balloons. The inflation time was set at $250 \mathrm{~ms}$ and the dwell time at 0 seconds, therefore, the esophageal stimuli had a duration of $\pm 250 \mathrm{~ms}$ (inflation + deflation time). Balloon pressures were determined using the ascending methods of limits approach. ${ }^{41}$ Participants rated the balloon distensions on an intensity visual analog scale ( $0=$ not intense, $10=$ very intense), after being instructed that these stimuli should not be painful. Balloon pressures corresponding to visual analog scale ratings of 4 (low-intensity stimulus, $S_{L}$ ) and 6 (high-intensity 

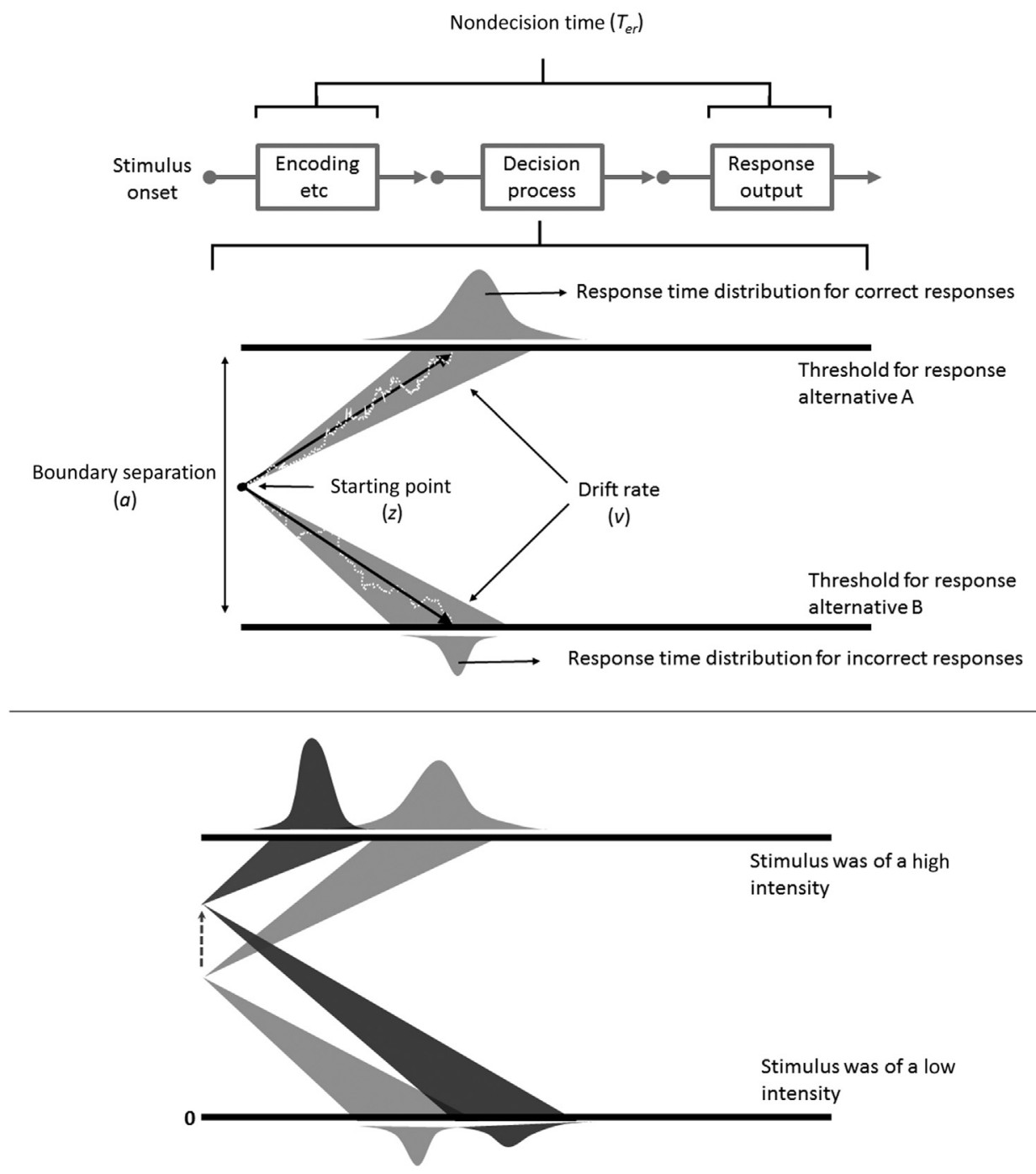

Figure 1. Overview of the DDM. Top panel: According to these models, perception is a stochastic process characterized by accumulation of noisy sensory input until a decision boundary is reached. ${ }^{35}$ DDMs are typically applied to 2 alternative forced choice data using separate RT distributions for correct and incorrect responses (ie, congruently accounting for mean RT, the variance of RT, and response accuracy) as input to estimate the model parameters. ${ }^{24,40}$ The decision process consists of the accumulation of evidence (ie, speed of information accumulation called drift rate [v]) from a certain starting point [ie, an a priori bias [z]) until 1 of 2 decision thresholds is reached and a response is executed. The distance between the orthogonal response thresholds is called the boundary separation (a) and represents the decision conservativeness (ie, how much evidence is required to make a decision). The nondecision component (Ter) is the time required for stimulus encoding and response execution. The white dotted lines represent a single accumulation trace of evidence, which is a noisy process. Bottom panel: The dark gray illustrates the effect of a shift in $z$ from the midpoint (no bias) toward the high-intensity threshold on the RTs distributions. Because of this shift in starting point, less evidence is required to categorize the stimulus as 'high' and the probability of high intensity percepts increases.

stimulus, $\mathrm{S}_{\mathrm{H}}$ ) were determined, and counterbalanced across participants as $\mathrm{CS}+$ and $\mathrm{CS}-$. Thereafter, to familiarize the subjects with the label corresponding to each stimulus, both pressure levels were presented and subjects were instructed to identify the high-intensity stimulus. If participants failed to correctly identify the $S_{H}$ during this task, both stimuli were recalibrated. Balloon pressures were recalibrated between the experimental phases. There were no differences in calibrated pressures between groups that were on the basis of CS allocation $\left(S_{L}: 15.37\right.$ pound force per square inch $[P S I ; S D=.96]$ vs 15.97 PSI [SD $=1.02$ ], $S_{H}: 18.1$ PSI [SD $=1.1$ ] vs 18.44 PSI [SD $=1.16]$, all with $P>.7$ ). We defined hypersensitivity as a difference of $<2$ PSI between first perception and pain threshold, and hyposensitivity when the required intensity for $\mathrm{S}_{\mathrm{H}}$ exceeded the maximum safe pressure of 35 PSI. When hypersensitivity or hyposensitivity was found, the session was terminated and the participant's data were excluded from the analysis.

\section{Electrical Stimulation}

Electrical stimulation was applied to the left ulnar styloid process using a commercially available electric stimulation device (Constant Current Stimulator, model DS7A; Digitimer, Hertfordshire, United Kingdom) delivering a train of thirty 2-ms monopolar square waveform pulses via 2 surface electrodes (V91-01, $8 \mathrm{~mm}$, Coulbourn Instruments, Holliston, MA) filled with $\mathrm{K}-\mathrm{Y}$ jelly (Johnson \& Johnson, New Brunswick, NJ). The stimulus intensity 
4 The Journal of Pain

corresponding to a rating of 8 on a visual analog scale ranging from 0 (no pain) to 10 (worst pain imaginable) was determined for each individual using the ascending methods of limits approach ${ }^{41}$ and then served as the US. The average stimulation intensity was $7.59 \mathrm{~mA}(\mathrm{SD}=3.8)$. After this initial US calibration, the esophageal stimuli were recalibrated. Before acquisition and each postacquisition phase, US intensity was recalibrated, if necessary, to ensure consistent pain levels throughout the experiment.

\section{Startle Probe}

The startle probe was a 50-ms burst of white noise with a peak of $105 \mathrm{dBA}$, which was presented binaurally through headphones (HD 418; Sennheiser, Wedemark, Germany).

\section{Procedure}

Upon arrival, participants read and signed the informed consent form. As part of another study, a 6minute electrocardiographic recording was performed before the actual experiment. Next, trained medical personnel inserted the esophageal probe. Despite the unpleasant nature of the probe insertion, most participants habituated within a few minutes (ie, they reported they barely perceived the deflated probe). Thereafter, participants were familiarized with the visceral sensations induced by balloon distension through presentation of several distensions of increasing intensity, until discomfort or pain was reported.

\section{Preacquisition Phase}

After calibration, 10 startle probes were presented to allow for habituation. ${ }^{3}$ Next, participants were instructed to categorize esophageal stimuli as 'high' or 'low' as quickly and as accurately as possible, by pressing 1 of 2 response buttons with their dominant hand. The allocation of the response buttons to the 2 categories (ie, 'high' or 'low') was counterbalanced between participants. To remind participants of the button allocation, the words 'high' and 'low' were shown on the computer screen in the appropriate locations, throughout the experiment. A total of $30 \mathrm{CS}+$ and $30 \mathrm{CS}$ - trials were presented. Intertrial intervals varied randomly between 7 and 12 seconds. Startle probes were presented 2 seconds before (in $10 \%$ of the trials) or 5.5 seconds after stimulus onset (in $30 \%$ of the trials). Trial types were presented in a pseudorandomized order with the restrictions of: 1 ) no more than 1 consecutive trial with a startle probe, and 2) no more than 2 consecutive trials with the same CS. The preacquisition phase was separated into 3 blocks ( 10 CS + and 10 CSper block), with a 3-minute break between blocks to minimize habituation or sensitization. During this break, the perceived intensities of the CS + and CS - were reassessed and the stimuli were adjusted accordingly.

\section{Acquisition Phase}

At this point, participants were instructed: 1) to continue to categorize the stimuli using the response
Biased Visceral Perception Through Fear Learning

buttons, 2) that for the remainder of the experiment, electrical stimulations could be presented in addition to the esophageal balloons, and 3 ) that if participants were attentive, they might be able to predict the occurrence of the electrical stimulus (US). During this phase, the $\mathrm{CS}+$ and the $\mathrm{CS}$ - were each presented 10 times, with $100 \%$ reinforcement of the CS + with the US. The US was presented 6 seconds after CS + onset (ie, trace conditioning). The $S_{L}$ was chosen as the $\mathrm{CS}+$ in half of the sample, whereas the $S_{H}$ was chosen as $\mathrm{CS}+$ in the other half.

\section{Postacquisition Phase}

This phase was identical to the preacquisition phase, except that the US was presented in $60 \%$ of the CS + trials (partial reinforcement, 6 of 10 trials per block) to prevent extinction, similar to Zaman et al. ${ }^{44}$

\section{Measures}

\section{GSR}

The GSR is considered to be under the control of the sympathetic branch of the autonomic nervous system that controls the activation of sweat glands and hence affects the conductive capacity of the skin. ${ }^{13}$ A sweating response is often observed after presentation of aversive stimuli. In this study, the GSR was recorded with LabLinc $\mathrm{V}$ (Lincoln, NB) $\mathrm{AgCl}$ electrodes (8 $\mathrm{mm}$ diameter, well depth $2 \mathrm{~mm}$ ) filled with $\mathrm{K}-\mathrm{Y}$ jelly (Johnson \& Johnson) and attached to the hypothenar palm of the nondominant hand, the skin of which had first been abraded. The interelectrode distance was $2.5 \mathrm{~cm}$. A Coulbourn Instruments skin conductance coupler (LabLinc v71-23) provided a constant $.5 \mathrm{~V}$ across the electrodes. The signal was digitized at $100 \mathrm{~Hz}$ throughout the study. GSRs were calculated by subtracting the maximum skin conductance level during the 2-second interval preceding balloon inflation from the maximum skin conductance level during the 6 seconds after stimulus onset. Responses below a value of $.05 \mu \mathrm{s}$ were considered to be nonresponses and set to 0 . Data were log transformed $(\log 10[1+\mathrm{GSR}])$ to normalize the distribution.

\section{Startle Eyeblink Reflex}

The startle eyeblink reflex is a frequently adopted psychophysiological index of covert defensive mobilization, because the strength of its amplitude is greater when the subcortical defensive fear network is activated. ${ }^{5,16}$ Orbicularis oculi electromyographic activity was recorded using three $\mathrm{Ag} / \mathrm{AgCl}$ electrodes (V91-02, $4 \mathrm{~mm}$; Coulbourn Instruments), according to the guidelines described by Blumenthal et al. ${ }^{3}$ To reduce interelectrode resistance, the skin was pretreated with a mild abrasive cream (body scrub, Inecto, United Kingdom). A Coulbourn Instruments isolated bioamplifier (v75-04) amplified the raw signal with a $13-\mathrm{Hz}$ high pass and $500-\mathrm{Hz}$ low pass bandpass filter. This signal was then rectified and smoothed with a time constant of 20 ms (Integrator V76-24; Coulbourn Instruments). Startle responses sampled at $1000 \mathrm{~Hz}$ were recorded from 
$200 \mathrm{~ms}$ before probe onset until $800 \mathrm{~ms}$ after probe onset. Startle amplitudes were calculated by subtracting the mean baseline value $(0-20 \mathrm{~ms}$ after probe onset) from the peak value found in the 21- to 175-ms time window after startle probe onset. Startle responses were visually inspected (offline) for artifacts (eg, spontaneous blinks) and rejected if necessary. Startle data from 12 participants were excluded because more than $30 \%$ of their startles were classified as nonresponses (ie, nonresponders). To reduce interindividual variation, startle amplitudes were transformed into T-scores and mean startle amplitudes were calculated per phase and CS. If a mean was on the basis of fewer than 3 observations, it was coded as a missing value ( $21 \%$ of the data cells). ${ }^{13}$

\section{Response Accuracy}

Response accuracies were on the basis of the total number of responses made, rather than on the number of stimuli presented, so as to account for trials during which participants did not respond (percentage of omitted responses, $\mathrm{S}_{\mathrm{L}}: 5.65 \%$ [SD $=10.9 \%$ ], $\mathrm{S}_{\mathrm{H}}: 3.24 \%$ $[S D=10.6 \%])$. Accuracies per phase for each CS were therefore defined as the total number of correct responses divided by the total number of responses for that CS and in that phase.

\section{RTS}

RTs were operationalized as the time from CS onset until a response button was pressed. Mean RTs were calculated separately for incorrect and correct responses per CS and phase.

\section{Software}

Stimulus presentation and data collection was performed using Affect 4.0 software, ${ }^{31}$ a 16 -bit data acquisition card (National Instruments, Austin, TX) and a Pentium-III computer (Intel, Santa Clara, CA). Physiological data were then processed offline, using PSPHA, ${ }^{10}$ for parameter extraction.

\section{Data Analysis}

As outlined in the introductory section, we expected differential fear learning to result in: 1) a greater increase in startle amplitudes from preacquisition to acquisition for the $\mathrm{CS}+$ than the $\mathrm{CS}-$, and 2) less habituation from preacquisition to acquisition for the CS + compared with the CS-, for the GSR data. GSR data and response accuracy were analyzed using a repeated measures analysis of variance (ANOVA) with CS (CS+/CS-) and phase (preacquisition/acquisition/postacquisition) as within subject factors and group ( $\mathrm{CS}+: \mathrm{S}_{\mathrm{L}} / \mathrm{CS}+: \mathrm{S}_{\mathrm{H}}$ ) as a between subjects factor. Because the data for startle responses as well as RTs contained missing responses, they were analyzed using mixed models, which provide a powerful and flexible approach to analyzing repeated measures data with missing data cells. ${ }^{2,29,38}$ For the startle data, an unstructured covariance matrix was preferred to its second best-fitting competitor (heterogenous Toeplitz: $\left.\chi^{2}{ }_{10}=40.22, P<.001\right)$. The model included CS
(CS+/CS-) and phase (preacquisition/acquisition/postacquisition) as within subject factors, group (CS + : $\mathrm{S}_{\mathrm{L}} / \mathrm{CS}+$ : $\mathrm{S}_{\mathrm{H}}$ ) as a between subject factor, and all interactions. For RT analyses see the Supplementary data. Greenhouse-Geiser corrections were applied for violations of sphericity when appropriate. Uncorrected degrees of freedom and corrected $P$ values are reported together with $\varepsilon$. Partial squared eta $\left(\eta_{\mathrm{p}}{ }^{2}\right)$ effect sizes are reported for the ANOVAs and post hoc tests, with small, medium, and large effects corresponding to values of $.0099, .0588$, and .1379 , according to Cohen. ${ }^{9}$ Note that no effect sizes are reported for the mixed models and post hoc tests within the model, because these models do not allow for estimation of effect sizes. In addition, degrees of freedom within these models are differentially estimated compared with regular repeated measures ANOVAs, because they rely on the Satterthwaite approximation. ${ }^{26}$ All post hoc contrasts were tested 2-tailed and corrected for multiple testing using the stepdown Bonferroni correction. All data analyses were performed using SPSS 20 (IBM Corp, Armonk, NY).

\section{Hierarchical DDM}

Parameters of the decision-making process were estimated with a hierarchical DDM, using a Python-based algorithm (http://ski.clps.brown.edu/hddm_docs; Wiecki et $\mathrm{al}^{40}$ ), on the basis of the RT distributions for correct and incorrect responses of each participant. Hierarchical models allow parameters from each individual to be drawn from a group distribution and for parameters to be estimated at either the group or individual level. Consequently, they require fewer data points per participant compared with the traditional diffusion models. ${ }^{36}$ For this analysis, the categorization data (RT and response accuracy) from only preacquisition and postacquisition were used. The following parameters (see Fig 1) of the DDM were modeled:

1) The speed at which evidence accumulates, called the 'drift rate';

2) A 'nondecision time' that combines the time required for stimulus encoding with the time required for execution of the response (ie, total RT excluding the time required for decision-making);

3) The 'boundary separation,' which is the distance between the 2 response thresholds; and

4) The point between the 2 decision thresholds at which evidence accumulation begins, the 'starting point' (z).

The model was set up such that drift rate and nondecision time were allowed to differ between phases (preacquisition, postacquisition) and between stimuli $\left(S_{L}\right.$, $\mathrm{S}_{\mathrm{H}}$ ), whereas boundary separation and starting point were allowed to differ between phases only. Intertrial variability was allowed for the nondecision component. Markov chain Monte-Carlo sampling methods were used to generate probability distributions for the parameters-the 'posterior distributions.' The reader is referred to http://ski.clps.brown.edu/hddm_docs for 
6 The Journal of Pain

technical details of this analytical approach. We opted to use noninformative priors, which are uniform distributions with equal probabilities across a range of parameter values. In total, 40,000 samples were generated and the first 5,000 were discarded because initial samples are considered unreliable because of random selection of initial values, and thinned by a factor 2 . The hierarchical DDM convergence diagnostics (all Rhats $<1.01$ ) indicated that Markov chain Monte-Carlo convergence was reached. On the basis of the assumption that outliers were due to different processes, ${ }^{25} \mathrm{RTs}$ \pm 2 SD and RTs $<200 \mathrm{~ms}$ from each participant's mean were removed (approximately $4.9 \%$ of each participant's data; SD of RTs $=1.45$ ) before model fitting. Hypothesis testing was performed on the basis of the overlap between the posterior distributions (ie, probability distribution across potential values for a given parameter) of the parameters of interest (drift rate, nondecision time, boundary separation, starting point) for 1 condition (eg, preacquisition) compared with another condition (eg, postacquisition) ${ }^{40}$ For example, we assessed the difference between the probability that the $z$ has a certain value during preacquisition and the probability that it has the same value during postacquisition (ie, the probability of overlap between the distributions of $z$ for preacquisition and postacquisition). Only if the probability of overlap between the distributions was $<2.5 \%$ did we infer a difference between both conditions-an approach similar to Wiech et al. ${ }^{39}$ The probability of overlap between the distributions of 2 conditions serves as a Bayesian analog to the classical $P$ value associated with a 2 -tailed test.

\section{Results}

\section{Manipulation Check}

\section{GSR: Repeated Measures ANOVA}

The $C S \times$ Phase $\times$ Group interaction $\left(F_{2,116}=7.26\right.$, $\left.P=.001, \eta_{p}{ }^{2}=.11\right)$ was significant, so we compared GSR for both groups, across phases, for the $\mathrm{CS}+$ relative to the CS-. Post hoc tests revealed that the difference between GSRs to the CS + and CS - developed differently across phases in the 2 different groups. Specifically, there was a greater reduction in the difference in GSRs between the CS + and CS - in the first group (with $\mathrm{S}_{\mathrm{L}}$ as $\mathrm{CS}+$ ) than in the second group (with $\mathrm{S}_{\mathrm{H}}$ as $\mathrm{CS}+)$ from preacquisition to acquisition $\left(\mathrm{F}_{1,58}=8.72\right.$, $\left.P=.005, \eta_{\mathrm{p}}{ }^{2}=.13\right)$ as well as to postacquisition $\left(F_{1,58}=17.32, P<.001, \eta_{p}{ }^{2}=.23\right.$; Fig 2$)$. Therefore, GSR to the CS+ habituated less from preacquisition onward compared with GSR to the CS--, in the first group only. Results of the full omnibus test are provided in the Supplementary material.

\section{Startle Eyeblink Reflex: Mixed Model}

Similar to the GSR analyses, the CS $\times$ Phase $\times$ Group interaction $\left(F_{2,37.07}=5.61, P=.007\right)$ was significant. Post hoc tests revealed that the first group (with $S_{L}$ as $\mathrm{CS}+$ ) showed a stronger increase in startle amplitudes for the $\mathrm{CS}+$ relative to the $\mathrm{CS}_{-}$, from preacquisition to acquisition, than the second group (with $\mathrm{S}_{\mathrm{H}}$ as $\mathrm{CS}+$ ) $\left(t_{32.95}=3.04, P=.01\right)$. Further, the first group showed a significantly greater decrease in startle amplitudes for
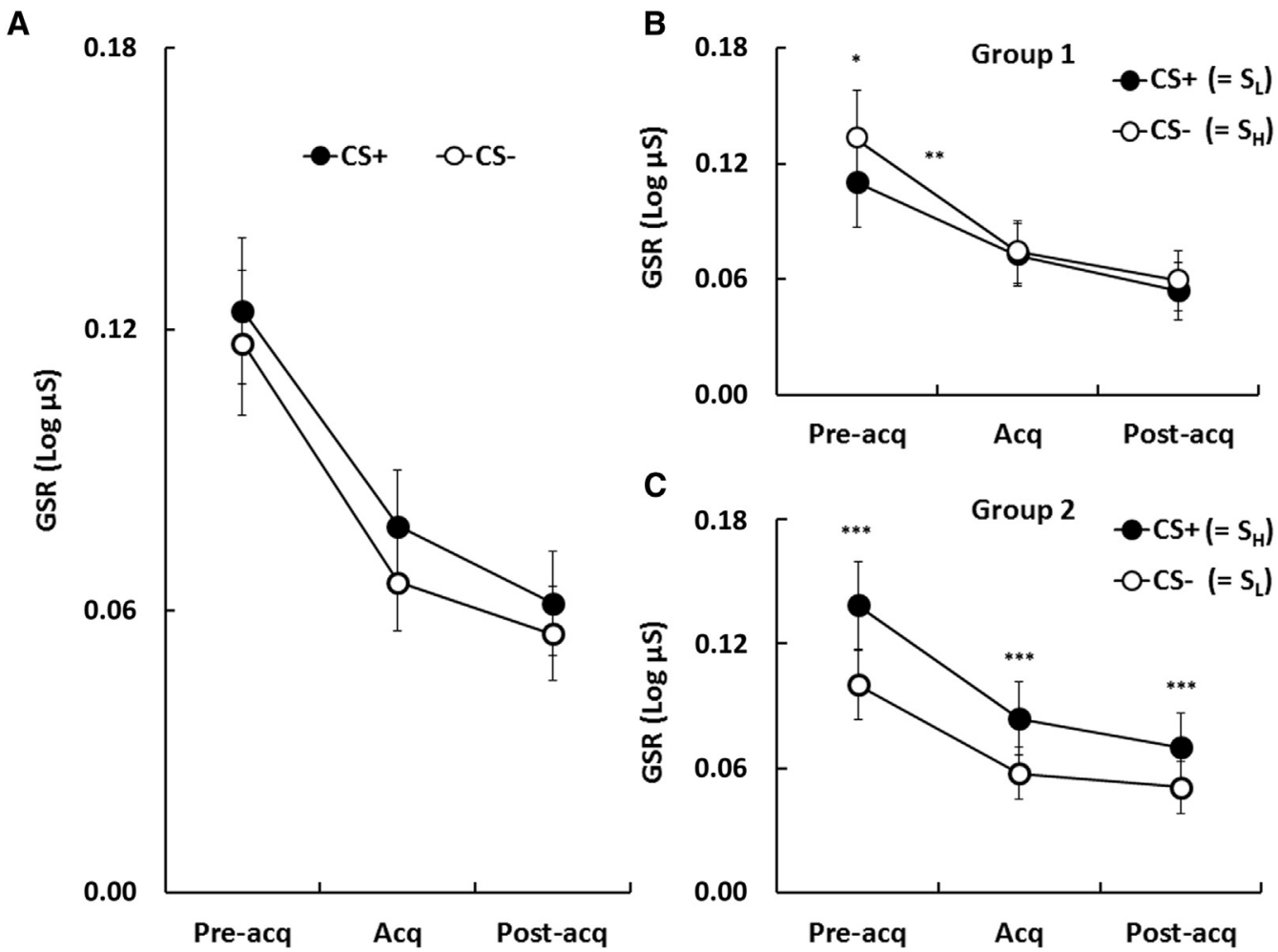

Figure 2. Mean GSR per phase. (A) GSRs across groups for the CS + and CS-. (B) GSRs for group 1 with $S_{L}$ as CS + . (C) GSRs for group 2 with $\mathrm{S}_{\mathrm{H}}$ as $\mathrm{CS}+$. Error bars represent standard errors of the mean. ${ }^{*} P \leq .05,{ }^{*} P<.01,{ }^{*} * P \leq .001$. Abbreviation: Acq, acquisition. 
Zaman et al

the $\mathrm{CS}$ + relative to the $\mathrm{CS}_{-}$, from acquisition to postacquisition than that shown by the second group $\left(t_{28.15}=3.12, P=.012 ; \mathrm{Fig} 3\right)$. In the second group, startle amplitudes to $\mathrm{CS}+$ as well as CS- were greater during acquisition than during pre- and postacquisition $\left(t_{33.83}=2.7, P=.011\right.$; Fig 3$)$. Therefore, we found a differential (CS+ vs CS-) increase in startle amplitudes from preacquisition to acquisition only in the group with the low-intensity stimulus as CS+. For results of the full omnibus tests, see the Supplementary material.

In sum, galvanic skin as well as startle eyeblink responses suggest increased fear responding to the $\mathrm{CS}+$ compared with the CS- from preacquisition to acquisition, in the group with the low-intensity stimulus as CS+ only. Therefore, we decided to run the planned DDM separately for each group, because differential fear learning was observed only in the group with the low-intensity stimulus as CS+.

\section{Behavioral Outcomes}

\section{Response Accuracy: Repeated Measured AN- OVA}

Again, we found a significant CS $\times$ Phase $\times$ Group interaction $\left(F_{2,114}=15.14, P<.001, \eta_{p}{ }^{2}=.21, \varepsilon=.88\right)$. Post hoc tests revealed that, in both groups, response accuracy for $S_{H}$ decreased from preacquisition to acquisition (first group: $F_{1,57}=11.81, P=.009, \eta_{p}^{2}=.17$; second group: $\mathrm{F}_{1,57}=7.78, P=.035, \eta_{\mathrm{p}}{ }^{2}=.12$ ), and only in the first group (with $\mathrm{S}_{\mathrm{H}}$ as $\mathrm{CS}-$ ) did it increase again
The Journal of Pain 7

during postacquisition (first group: $F_{1,57}=15.26$, $P=.006, \eta_{p}{ }^{2}=.21$; second group: $F_{1,57}=2.47, P=.4$ ). Response accuracies for $S_{L}$ did not change from preacquisition to acquisition in either group ( $P$ values $>.8$ ), whereas, from acquisition to postacquisition, they decreased in the second group only (with $S_{L}$ as $C S-$; first group: $F_{1,57}=2.39, P=.4$; second group: $F_{1,57}=8.37$, $\left.P=.032, \eta_{\mathrm{p}}{ }^{2}=.13\right)$. In both groups, response accuracies for $S_{L}$ decreased from preacquisition to postacquisition $\left(F_{1,57}=8.96, P=.032, \eta_{p}^{2}=.14 ; F_{1,57}=9.11, P=.032\right.$, $\eta_{\mathrm{p}}^{2}=.14$; Fig 4).

\section{Hierarchical DDM}

\section{Group 1: $\mathrm{S}_{\mathrm{L}}$ as CS+}

Fig 5 shows that the group that showed differential fear learning (first group; $S_{L}$ as $C S+$ ), showed a shift in the starting point of their decision-making process toward the high intensity threshold $(P=.021)$, indicating an a priori decision-making bias that favors the categorization of stimuli as high-intensity sensations. As a consequence, for this group, even before a stimulus is delivered, less evidence is required to reach the high intensity threshold compared with the low intensity threshold. Fig 5 also shows that the drift rate for $S_{H}$ was significantly lower than the drift rate for $S_{L}$ during pre- as well as postacquisition ( $P$ values $<.001$ ), indicating slower accumulation of evidence in favor of $\mathrm{S}_{\mathrm{H}}$. There was no change in drift rate, nondecision time or
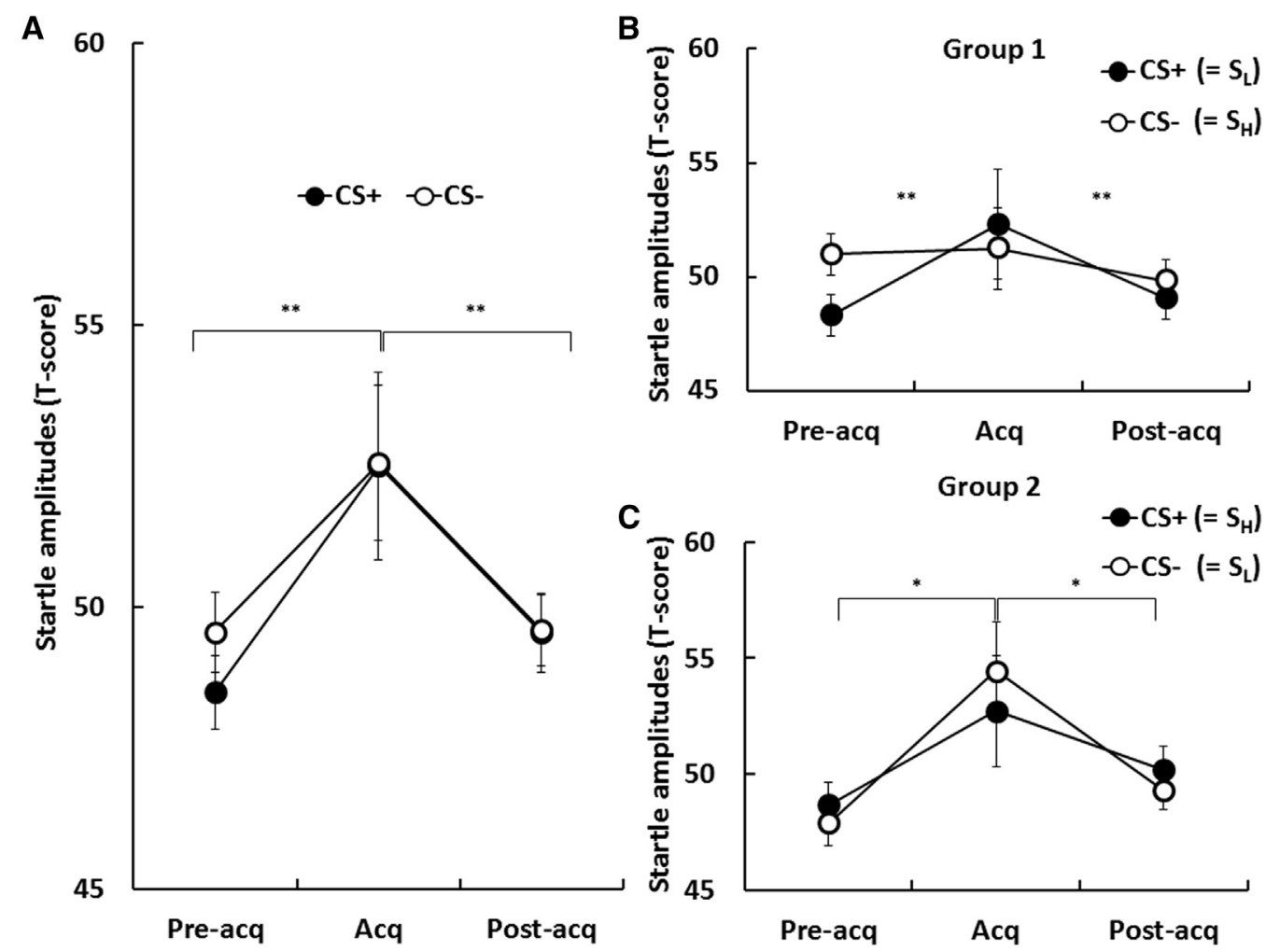

Figure 3. Mean startle eyeblink amplitudes. (A) Startle amplitudes across groups for the CS + and CS-. (B) Startle amplitudes for group 1 with $\mathrm{S}_{\mathrm{L}}$ as $\mathrm{CS}+$. (C) Startle amplitudes for group 2 with $\mathrm{S}_{\mathrm{H}}$ as $\mathrm{CS}+$. Error bars represent standard errors of the mean. ${ }^{*} P \leq .05,{ }^{*} P<.01$. Abbreviation: Acq, acquisition. 
A

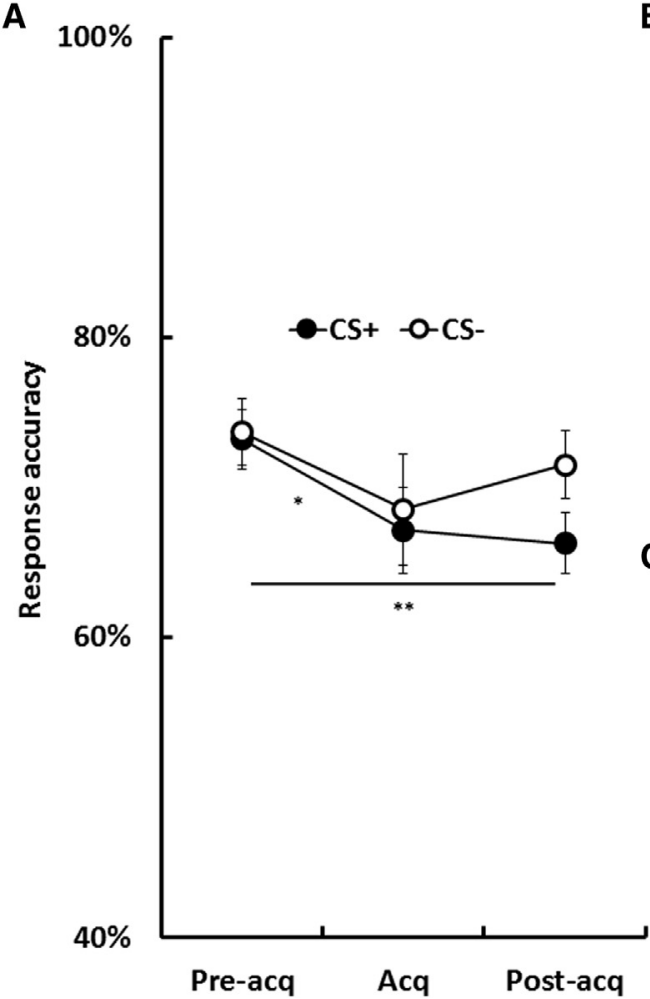

B
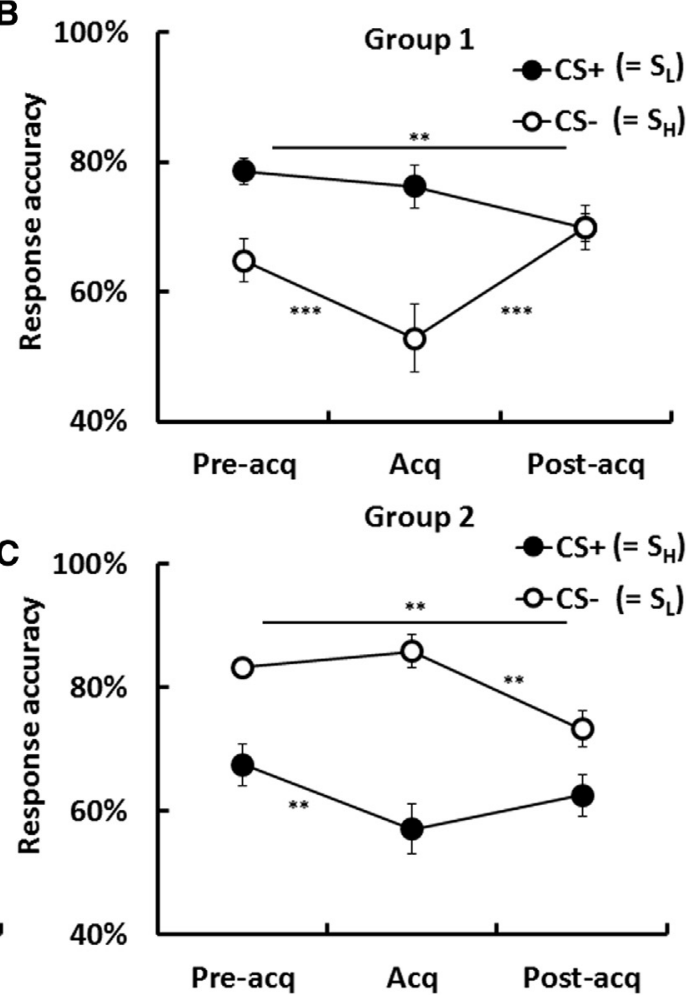

Figure 4. Mean response accuracies. (A) Response accuracies across groups for the $\mathrm{CS}+$ and $\mathrm{CS}-$. (B) Response accuracies for group 1 with $\mathrm{S}_{\mathrm{L}}$ as $\mathrm{CS}+$. (C) Response accuracies for group 2 with $\mathrm{S}_{\mathrm{H}}$ as $\mathrm{CS}+$. Error bars represent standard errors of the mean. ${ }^{\star} P \leq .05$, $* * P<.01$, $* * * P \leq .001$. Abbreviation: Acq, acquisition.

boundary separation from pre- to postacquisition $(P$ values $\geq .2)$.

\section{Group 2: $\mathrm{S}_{\mathrm{H}}$ as $\mathrm{CS}+$}

For the group that failed to demonstrate differential fear learning (second group; $\mathrm{S}_{\mathrm{H}}$ as $\mathrm{CS}+$ ), there was no shift in starting point from pre- to postacquisition, nor was there any change in drift rate or nondecision time (all $P$ values $>.4$ ). A trend for smaller boundary separations from pre- to postacquisition emerged $(P=.045)$. A significantly slower drift rate was observed for $S_{H}$ compared with $S_{\mathrm{L}}$ during pre- as well as postacquisition $(P$ values $<.001)$.

\section{Discussion}

The current study used healthy volunteers to investigate how learning to fear nonpainful visceral sensations influences the decision-making processes that underlies the perception of the intensity of visceral sensations. The differential conditioning paradigm used 2 nonpainful esophageal balloon distensions of different intensities as CSs and a painful electrical US, in combination with a 2-alternative forced choice categorization task. Differential fear learning was shown in only 1 group. Using a DDM, fear learning was found to introduce a decision-making bias, such that less sensory evidence was required for participants to perceive a stimulus as being of high intensity. In other words, fear learning shifted the starting point for the decision-making process toward the high-intensity threshold.

\section{Fear Learning}

Our data show that the conditioning procedure did not lead to differential fear learning when we analyzed data across both groups (ie, those with the low-intensity stimulus $\left[\mathrm{S}_{\mathrm{L}}\right]$ as $\mathrm{CS}+$ and those with the high-intensity stimulus $\left[\mathrm{S}_{\mathrm{H}}\right]$ as $\mathrm{CS}+$ ). However, separate analyses according to group (following up on significant 3-way interactions) showed differential fear learning in the former but not the latter group, suggesting a modulatory effect of stimulus intensity on fear learning. GSR as well as startle data indicate that differential fear learning was established in only the group for which the $S_{\mathrm{L}}$ was used as the CS + . This resembles observations in a previous study on differential fear conditioning that used different breathing loads (ie, breathing through resistors that induce a feeling of having to work for one's breath) as CSs and a breathing occlusion as US. ${ }^{28}$ In that study, participants learned to expect the US after the CS + , but only if they received the low-intensity load as $\mathrm{CS}+$; those who received the high-intensity load as CS+ did not show evidence of learning, as indexed by expectancy. The study also tested generalization of responses to a range of different intensity loads 24 hours later. Again, the typical decline in response strength as physical similarity from the CS + decreases $^{12}$ was observed only in the group with the low-intensity load as CS+. In the group with the high-intensity load as CS + , a flat generalization gradient was found, suggesting fear acquisition to $\mathrm{CS}+$ as well as CS-. The authors attributed their differential group effect to preexisting differences in US expectancy for the low- and the high-intensity CSs during 
A
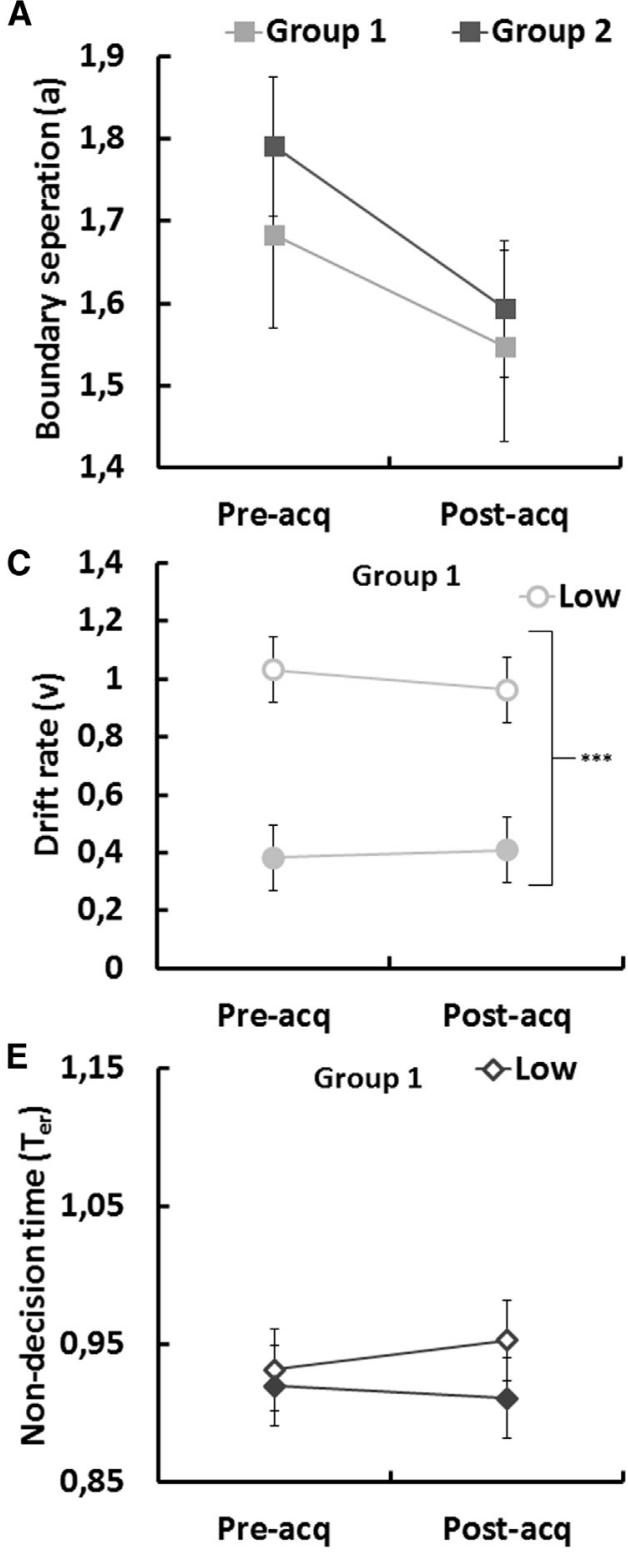

B
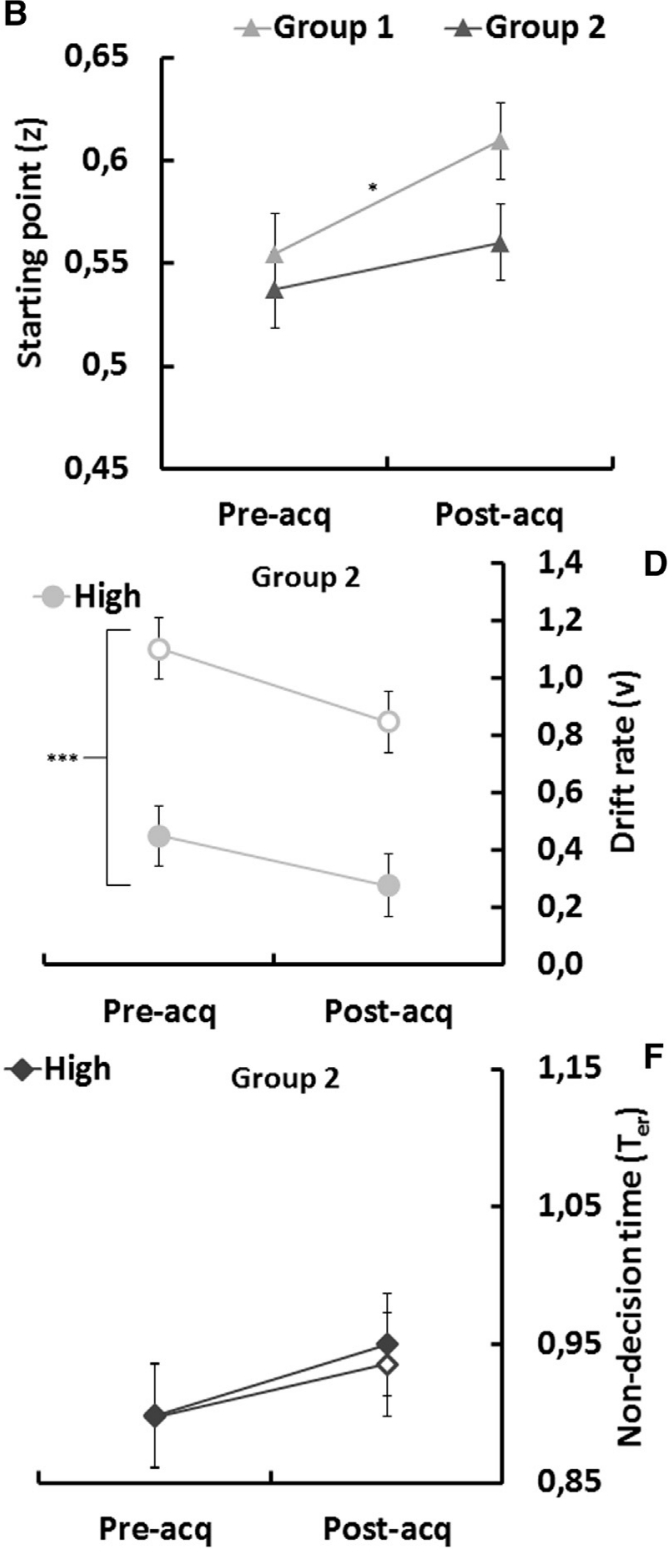

$1,15 F$

0,05

Figure 5. Drift diffusion parameter estimates. Population average of boundary separation (a) (A) and starting point ( $z$ ) (B) per group and phase. The population average drift rates $(v)$ for $S_{L}$ as well as $S_{H}$ per group (C: group 1; D: group 2) and nondecision time (Ter)for $S_{L}$ as well as $S_{H}$ per group (E: group 1; F: group 2) and phase. Error bars represent standard deviation of the group posterior. ${ }^{*} P \leq .05$, $\star * * P \leq .001$. Abbreviation: Acq, acquisition.

preacquisition. ${ }^{28}$ However, the current study's response accuracy data suggest an alternative explanation for the findings of both studies. As shown in Fig 4, the accuracy for only $S_{H}$ (but not $S_{L}$ ) decreased considerably during acquisition. This decrease may have affected participants' subsequently perceived CS-US contingencies differently in each group. In both groups a $100 \%$ reinforcement rate was adopted, but in the group with $\mathrm{S}_{\mathrm{H}}$ as $\mathrm{CS}+$ (second group), misperception of a $\mathrm{S}_{\mathrm{H}}$ trial as being $S_{L}$ would have resulted in the perception of $S_{H}$ as well as $S_{L}$ as predictive of the US. Consequently, $S_{H}$ as well as $S_{L}$ would be expected to elicit fear. In contrast, misperception of an $S_{H}$ trial as $S_{L}$ should not affect safety learning in the group with $\mathrm{S}_{\mathrm{L}}$ as $\mathrm{CS}+$ (as confirmed by the lack of startle potentiation for the CS- during acquisition). Further, in this group, a misidentification of a CS- as a CS + in the absence of the US would only decrease the perceived reinforcement rate of the $\mathrm{CS}+$, but would never violate the rule that the CS- predicts the absence of the US. Therefore, taking the perceived rather than the physical intensities of stimuli into account provides an alternative explanation for the observed group differences. Future experiments therefore need to control for such potential influences by assessing stimulus perception as well as fear responses. ${ }^{32}$

\section{Fear Learning Induced a Decision-Making Bias}

As expected, no changes were observed in the nondecision component, drift rate, or boundary separation of the decision-making process after fear learning. Only in 


\section{The Journal of Pain}

the group that showed differential fear learning did we observe a shift in starting point of the decision-making processes. However, contrary to our expectations, it did not shift toward the CS + threshold (ie, toward the categorization of the stimulus intensity as 'low'), but toward the high-intensity threshold. Thus, the distance between starting point and the high-intensity threshold became smaller, indicating that less sensory input needed to accumulate to reach this threshold. This shift suggests that an a priori decision-making bias developed so that the high-intensity percept was favored before any sensory input-in other words, less sensory input was required to categorize a visceral stimulus as intense. As such, fear learning may foster hypersensitivity and thereby contribute to pathological states such as hyperalgesia-perhaps due to a gradual spilling over of the characteristics of a painful US to the CS (for review, see Zaman et $a^{43}$ ). A similar shift in starting point was reported by Wiech et al, ${ }^{39}$ who combined a predictive cueing paradigm with a pain categorization task. After presentation of a cue, subjects received either a high or low painful stimulus and categorized each stimulus as high or low. In the first experiment, in which either a high pain cue or a noninformative cue was presented, the starting point shifted toward the threshold of the high painful stimulus when subjects expected the high pain stimulus. In the second experiment, which also included a low pain cue, a similar shift in starting point toward the low painful stimulus was observed. ${ }^{39}$ Their and our findings suggest that not only expectations but also motivational value acquired through fear learning can strongly bias (pain) perception, albeit to the highintensity stimulus rather than the $\mathrm{CS}+$. Interestingly, in our study the acquired differential startle responses disappeared during postacquisition, but categorization data (RTs and response accuracy) from pre- and postacquisition showed changes in perceptual decisionmaking. Hence, the current findings question the notion that a fearful state is a prerequisite to induce perceptual alterations. The same idea was called into question by a study that used odors as CSs: fear learning improved odor detection, but the extent of improvement did not relate to the degree of expressed fear. ${ }^{1}$ Similarly, degraded tone discrimination has been observed for tones associated with loss and those associated with reward, although to different degrees. ${ }^{27}$ These findings suggest that associative learning triggers perceptual alterations that may not depend upon the concurrent expression of fear.

\section{Clinical Implications and Limitations}

Our findings add further evidence to a growing body of research on interoceptive fear conditioning that shows that a wide range of interoceptive sensations can come to elicit stronger fear responses after association with aversive events, $7,20,21,28,42$ and that such learning processes can influence our perception of those sensations. ${ }^{43,44}$ Although the use of interoceptive CSs deviates from most conditioning work where neutral, exteroceptive
Biased Visceral Perception Through Fear Learning

CSs (eg, picture of a geometric shape) are used, we argue that visceral CSs better reflect clinical scenarios in which patients show a preoccupation with and fear of visceral sensations. ${ }^{15}$ The additive value of interoceptive compared with exteroceptive CSs for research on clinical disorders has been reported elsewhere., 21,28 The current study also shows that fear learning can be reliably inferred on the basis of the response changes to CS+ and CS- over time, which is similar to previous work with different intensity $\mathrm{CSs}^{28}$ We furthermore showed that benign visceral sensations can come to elicit fear despite a delay between these sensations and the experience of pain. This is particularly relevant to irritable bowel syndrome patients whose abdominal pain or cramps may commence some time after neutral (ie, non-noxious) sensations related to the digestive processes.

Despite these intriguing findings, some limitations should be acknowledged. The use of a painful electrical US may limit the ecological validity of our findings for functional gastrointestinal disorders. This was a practical decision: an interoceptive US would have placed undue strain on the esophagus, because of the large number of stimuli presented. Furthermore, simple between subject conditioning paradigms have shown successful fear learning to interoceptive CSs with interoceptive as well as exteroceptive USs. ${ }^{7,42,44}$

The decrease in categorization accuracy for $S_{H}$ from preacquisition to acquisition, regardless of $\mathrm{CS}$ allocation, was unexpected and unexplained. We speculate that the sudden presentation of the painful exteroceptive US during acquisition might have shifted attention from the processing of interoceptive stimulus to the more prominent exteroceptive stimulus (ie, hypervigilance). Such hypervigilance toward the painful US would have diverted attention away from the visceral sensations, possibly impairing accuracy. ${ }^{34}$

\section{Conclusions}

We showed that fear learning biased perceptual decision-making about the intensity of visceral sensations, such that sensations were more likely to be perceived as more intense, before any sensory input. This lines up with previous work linking GSA to visceral hypersensitivity and increased symptom reporting. ${ }^{14,15}$ Consequently, our findings suggest that such hypersensitivity in gastrointestinal patients might be partly driven by altered perception of visceral input as a result of learning to fear innocuous visceral stimuli.

\section{Acknowledgments}

The authors thank Madelon Peters for her assistance in helping them to revise the manuscript.

\section{Supplementary Data}

Supplementary data related to this article can be found at http://dx.doi.org/10.1016/j.jpain.2017.04.011. 


\section{References}

1. Åhs F, Miller SS, Gordon AR, Lundström JN: Aversive learning increases sensory detection sensitivity. Biol Psychol 92:135-141, 2013

2. Blackwell E, de Leon CF, Miller GE: Applying mixed regression models to the analysis of repeated-measures data in psychosomatic medicine. Psychosom Med 68:870-878, 2006

3. Blumenthal TD, Cuthbert BN, Filion DL, Hackley $\mathrm{S}$, Lipp OV, van Boxtel A: Committee report: Guidelines for human startle eyeblink electromyographic studies. Psychophysiology 42:1-15, 2005

4. Bogacz R, Brown E, Moehlis J, Holmes P, Cohen JD: The physics of optimal decision making: A formal analysis of models of performance in two-alternative forced-choice tasks. Psychol Rev 113:700-765, 2006

5. Bradley MM, Lang PJ: Affective reactions to acoustic stimuli. Psychophysiology 37:204-215, 2000

6. Cavanagh JF, Wiecki TV, Kochar A, Frank MJ: Eye tracking and pupillometry are indicators of dissociable latent decision processes. J Exp Psychol Gen 143:1476-1488, 2014

7. Ceunen E, Zaman J, Weltens N, Sarafanova E, Arijs V, Vlaeyen JW, Van Oudenhove L, Van Diest I: Learned fear of gastrointestinal sensations in healthy adults. Clin Gastroenterol Hepatol 14:1552-1558.e2, 2016

8. Coffin B, Dapoigny M, Cloarec D, Comet D, Dyard F: Relationship between severity of symptoms and quality of life in 858 patients with irritable bowel syndrome. Gastroenterol Clin Biol 28:11-15, 2004

9. Cohen J: The statistical power of abnormal-social psychological research: A review. J Abnorm Soc Psychol 65:145-153, 1962

10. de Clercq A, Verschuere B, de Vlieger P, Crombez G: Psychophysiological analysis (PSPHA): A modular script-based program for analyzing psychophysiological data. Behav Res Methods 38:504-510, 2006

11. Elsenbruch S: Abdominal pain in irritable bowel syndrome: A review of putative psychological, neural and neuro-immune mechanisms. Brain Behav Immun 25: 386-394, 2011

12. Ghirlanda S, Enquist M: A century of generalization. Anim Behav 66:15-36, 2003

13. Dawson ME, Schell AM, Fillion DL: The Electrodermal System. In: Cacioppo JT, Tassinary LG, Berntson G (eds): Handbook of Psychophysiology. Cambridge, Cambridge University Press 159-181, 2007

14. Labus JS, Bolus R, Chang L, Wiklund I, Naesdal J, Mayer EA, Naliboff BD: The Visceral Sensitivity Index: Development and validation of a gastrointestinal symptomspecific anxiety scale. Aliment Pharmacol Ther 20:89-97, 2004

15. Labus JS, Mayer EA, Chang L, Bolus R, Naliboff BD: The central role of gastrointestinal-specific anxiety in irritable bowel syndrome: Further validation of the visceral sensitivity index. Psychosom Med 69:89-98, 2007

16. Lang PJ, Davis M, Ohman A: Fear and anxiety: Animal models and human cognitive psychophysiology. J Affect Disord 61:137-159, 2000

17. Lipp OV: Human fear learning: Contemporary procedures and measurement. In: Craske MG, Hermans D,
Vansteenwegenin D (eds): Fear Learning From Basic Process to Clinical Implications. Washington, DC, American Psychological Association, 2006, pp 39-52

18. Mulder MJ, Wagenmakers EJ, Ratcliff R, Boekel W, Forstmann BU: Bias in the brain: A diffusion model analysis of prior probability and potential payoff. J Neurosci 32: 2335-2343, 2012

19. Nyrop KA, Palsson OS, Levy RL, Von Korff M, Feld AD, Turner MJ, Whitehead WE: Costs of health care for irritable bowel syndrome, chronic constipation, functional diarrhoea and functional abdominal pain. Aliment Pharmacol Ther 26: 237-248, 2007

20. Pappens M, Smets E, Vansteenwegen D, Van Den Bergh O, Van Diest I: Learning to fear suffocation: A new paradigm for interoceptive fear conditioning. Psychophysiology 49:821-828, 2012

21. Pappens $M$, Van den Bergh $O$, Vansteenwegen $D$, Ceunen E, De Peuter S, Van Diest I: Learning to fear obstructed breathing: Comparing interoceptive and exteroceptive cues. Biol Psychol 92:36-42, 2013

22. Pavlov IP: Conditioned Reflexes: An Investigation of the Physiological Activity of the Cerebral Cortex. Oxford, England, Oxford University Press, 1927

23. Ratcliff R: A diffusion model account of response time and accuracy in a brightness discrimination task: Fitting real data and failing to fit fake but plausible data. Psychon Bull Rev 9:278-291, 2002

24. Ratcliff R, McKoon G: The diffusion decision model: Theory and data for two-choice decision tasks. Neural Comput 20:873-922, 2008

25. Ratcliff R, Tuerlinckx F: Estimating parameters of the diffusion model: Approaches to dealing with contaminant reaction times and parameter variability. Psychon Bull Rev 9:438-481, 2002

26. Satterthwaite FE: An approximate distribution of estimates of variance components. Biometrics 2:110-114, 1946

27. Schechtman E, Laufer O, Paz R: Negative valence widens generalization of learning. J Neurosci 30:10460-10464, 2010

28. Schroijen M, Pappens M, Schruers K, Van den Bergh O, Vervliet B, Van Diest I: Generalization of fear to respiratory sensations. Behav Ther 46:611-626, 2015

29. Snijders TA, Bosker RJ: Multilevel Analysis: An Introduction to Basic and Advanced Multilevel Modeling, 2nd ed. London, Sage Publishers, 2012

30. Spiller R, Garsed K: Postinfectious irritable bowel syndrome. Gastroenterology 136:1979-1988, 2009

31. Spruyt A, Clarysse J, Vansteenwegen D, Baeyens $F_{4}$ Hermans D: Affect 4.0: A free software package for implementing psychological and psychophysiological experiments. Exp Psychol 57:36-45, 2010

32. Struyf D, Zaman J, Vervliet B, Van Diest I: Perceptual discrimination in fear generalization: Mechanistic and clinical implications. Neurosci Biobehav Rev 59:201-207, 2015

33. Talley NJ: Functional gastrointestinal disorders as a public health problem. Neurogastroenterol Motil 20(Suppl 1): 121-129, 2008

34. van Ede F, de Lange FP, Maris E: Attentional cues affect accuracy and reaction time via different cognitive and neural processes. J Neurosci 32:10408-10412, 2012 


\section{The Journal of Pain}

35. Van Oudenhove L, Aziz Q: The role of psychosocial factors and psychiatric disorders in functional dyspepsia. Nat Rev Gastroenterol Hepatol 10:158-167, 2013

36. Vandekerckhove J, Tuerlinckx F, Lee MD: Hierarchical diffusion models for two-choice response times. Psychol Methods 16:44-62, 2011

37. Weltens N, Schaub N, Van Oudenhove L, Ly H, Aziz Q, Tack J, Coen S: Positive and negative mood modulate esophageal pain perception in health. Gastroenterology 144:S556, 2013

38. West BT: Analyzing longitudinal data with the linear mixed models procedure in SPSS. Eval Health Prof 32: 207-228, 2009

39. Wiech $K$, Vandekerckhove J, Zaman J, Tuerlinckx $F$, Vlaeyen JWS, Tracey I: Influence of prior information on pain involves biased perceptual decision-making. Curr Biol 24:679-681, 2014
Biased Visceral Perception Through Fear Learning

40. Wiecki TV, Sofer I, Frank MJ: HDDM: Hierarchical Bayesian estimation of the Drift-Diffusion Model in Python. Front Neuroinform 7:14, 2013

41. Yarnitsky D, Sprecher E, Zaslansky R, Hemli JA: Heat pain thresholds: Normative data and repeatability. Pain 60: 329-332, 1995

42. Zaman J, De Peuter S, Van Diest I, Van den Bergh O, Vlaeyen JW: Interoceptive cues predicting exteroceptive events. Int J Psychophysiol 109:100-106, 2016

43. Zaman J, Vlaeyen JW, Van Oudenhove L, Wiech K, Van Diest I: Associative fear learning and perceptual discrimination: A perceptual pathway in the development of chronic pain. Neurosci Biobehav Rev 51:118-125, 2015

44. Zaman J, Weltens N, Ly HG, Struyf D, Vlaeyen JW, Van den Bergh O, Wiech K, Van Oudenhove L, Van Diest I: Influence of interoceptive fear learning on visceral perception. Psychosom Med 78:248-258, 2016 